Making your skin crawl: The role of tactile sensitivity in disease avoidance

David Francis Hunt, Grace Cannell, Nicholas A. Davenhill, Stephanie A. Horsford, Diana S.

Fleischman, and Justin H. Park

\author{
Author Note \\ David Francis Hunt, School of Experimental Psychology, University of Bristol, Bristol, \\ United Kingdom \\ Grace Cannell, Department of Psychology, University of Portsmouth, Portsmouth, United \\ Kingdom \\ Nicholas A. Davenhill, Department of Psychology, University of Bath, Bath, United \\ Kingdom \\ Stephanie A. Horsford, Department of Psychology, University of Bath, Bath, United \\ Kingdom \\ Diana S. Fleischman, Department of Psychology, University of Portsmouth, Portsmouth, \\ United Kingdom \\ Justin H. Park, School of Experimental Psychology, University of Bristol, Bristol, United \\ Kingdom
}

Correspondence concerning this article should be addressed to David Francis Hunt, School of Experimental Psychology, University of Bristol, 12a Priory Road, Bristol, BS8 1TU. Email: dh0191@bristol.ac.uk 


\begin{abstract}
Mounting evidence indicates that animals, including humans, have evolved a behavioral disease-avoidance system designed to facilitate the detection and avoidance of sources of pathogens, and that this system interacts with physiological defenses. The skin acts as an important anatomical barrier, yet little research has investigated the role of tactile sensitivity in disease avoidance. Increased tactile sensitivity in the presence of potential sources of pathogens may facilitate prophylactic behaviors such as self-grooming. Across multiple studies, we tested the hypothesis that the induction of disgust - the key emotion underlying disease avoidance — may lead to greater tactile sensitivity compared to control conditions. A nonsignificant trend was found in the pilot study, which was replicated (and found to be significant) in Studies 1 and 2. To our knowledge, these results are the first to demonstrate disgust-induced changes in tactile sensitivity, and they contribute to the growing literature on the integrated evolved defenses against infectious disease.
\end{abstract}

Keywords: behavioral immune system, disease avoidance, disgust, threat, tactile sensitivity 


\section{Making your Skin Crawl: The Role of Tactile Sensitivity in Disease Avoidance}

Parasites and pathogens pose one of the greatest threats to human survival and have played a central role in shaping the evolution of human physiology and behavior (Prokop \& Fedor, 2013; Wolfe, Dunavan, \& Diamond, 2007). While animals, including humans, have evolved a highly complex set of physiological mechanisms (i.e., the immune system) to manage infections (Hart 1993; Parham, 2009; Schaller, 2011), diseases and immune responses can be highly costly. Consequently, it has been proposed that animals have also evolved a behavioral immune system (BIS) that is designed to detect and facilitate avoidance of sources of infectious disease (Curtis, de Barra, \& Aunger, 2011; Oaten, Stevenson, \& Case, 2009; Schaller \& Park, 2011). The BIS consists of psychological mechanisms that are attuned to perceptual cues associated with pathogens and that deploy aversive emotions, cognitions, and behavioral responses. A burgeoning literature has documented the characteristics of the BIS and its wide-ranging implications (for a review, see Murray \& Schaller, 2016). There are two noteworthy characteristics of the BIS. The first is that it is functionally flexible, deploying heightened responses when perceived threat of infection is greater (e.g., exposure to stimuli associated with disease). The second is that disgust is the central emotion driving disease avoidance in humans (e.g., stimuli associated with disease elicit disgust and activate the BIS; Curtis et al., 2011; Schaller \& Park, 2011).

In some ways, the BIS can be seen as a component of a broader system for combating disease, as conceptualized by researchers in the field of psychoneuroimmunology (Clark \& Fessler, 2014). Indeed, growing evidence demonstrates that the "behavioral" and the "physiological" components are closely intertwined. This is illustrated by the role of disgust, the key emotion associated with disease avoidance. Consistent with Oaten et al.'s (2009) suggestion that immunological functioning could be activated via the perception of disgustevoking stimuli, exposure to disgust-evoking stimuli has been found to promote white blood 
cell responses (Schaller, Miller, Gervais, Yager, \& Chen, 2010), produce greater oral inflammatory responses (Stevenson, Hodgson, Oaten, Barouei, \& Case, 2011), and increase body temperature (Stevenson, Hodgson, Moussavi, Langberg, Case, \& Barouei, 2012). Conversely, people who have recently been ill (and have had their physiological system activated) have been found to exhibit heightened disease-avoidance tendencies (Miller \& Maner, 2011).

Many diseases are transmitted by microparasites and parasite vectors (e.g., flies, fleas, and ticks), often through skin lesions. Such disease threats have long posed a selection pressure on humans (Carter \& Mendis, 2002; Gonçalves, Araújo, \& Ferreira, 2003). Vector disease transmission can occur via processes such as mite infestations associated with scabies (Fuller, 2013) and mosquito bites associated with malaria (Autino, Noris, Russo, \& Castelli, 2012). Mechanical transmission from parasites found on the bodies of insects include gastroenteritis and trachoma (Graczyk, Knight, Gilman, \& Cranfield, 2001), and Escherichia coli (Echeverria, Harrison, Tirapat, \& McFarland, 1983).

Due to the prominence of skin-transmitted diseases, the skin is expected to play a central role in disease avoidance. Not only does the skin provide a physical barrier against the intrusion of pathogens (Madison, 2003), it also secretes antibodies against parasites (Hosoi, 2006). Far more than just a passive barrier, the skin has been implicated as part of the immune system, providing multiple epidermal and dermal cell populations that respond rapidly to any contact between the organism and its environment (Williams \& Kupper, 1996).

As the skin is implicated with the immune system and has specific function to deal with parasite load, it is likely that there will be a behavioral component that is specifically designed to complement these functions of detecting and avoiding disease threat. Animal and insect studies support the role of skin sensitivity for detection of disease threat; resultant behaviors such as scratching and oral grooming serve behavioral avoidance of disease (Hart, 
1990; de Roode \& Lefèvre, 2012). Four different ant species have been found to upregulate grooming in response to detecting contamination on nest mates (Tranter, Lefèvre, Evison, \& Hughes, 2014). For rats, the removal and minimization of ectoparasites is resource intensive: between 8 and 30 percent of their evaporative water loss is due to oral grooming (Bolles, 1960). And female antelopes have been observed to engage in self-grooming and scratching approximately 2,000 times during a 12-hour period (Hart \& Hart, 1988). Moreover, experiments found increased ectoparasite infestation among mice and cows who were unable to self-groom orally or scratch (Bell, Jellison, \& Owen, 1962; Bennett, 1969).

Humans also possess hygiene behaviors that are associated with disease avoidance (Curtis et al., 2011). Specific behaviors such as washing and scrubbing with different products (i.e., soaps and shower gels) are a form of self-grooming, as are more basic behaviors, such as scratching ${ }^{1}$ and hand wiping. Indeed, a recent study has shown that selfgrooming increases following exposure to disgust-evoking stimuli (Prokop, Fančovičová, \& Fedor, 2014). Increased skin sensitivity may be a key precursor to self-grooming and is particularly important for minimizing skin transmitted disease threat. Both early and accurate detection of disease threat allows the host to either avoid the associated costs altogether (complete removal) or mitigate the associated costs (e.g., minimizing infestation). Therefore, in line with the notion of functional flexibility (Murray \& Schaller, 2016), it can be hypothesized that tactile sensitivity may be heightened in the presence of cues that elicit disgust and suggest increased threat of disease. Detection subsequently promotes avoidance behavior with the function of either removing or at least minimizing the costs associated with contamination.

\section{The present studies}

We conducted multiple studies to test the hypothesis that exposure to disgust-evoking stimuli may increase tactile sensitivity. The first was a pilot study, which served as a 
feasibility study. Study 1 aimed to provide a more robust test by using a double-blind procedure and adequately powered. Study 2 attempted to replicate Study 1, using different disgust-evoking stimuli, and with an additional disease-irrelevant threat condition.

\section{Pilot study}

\section{Participants and design}

Forty undergraduate students ( 24 women, 16 men; mean age $=19.90$ years, $S D=$ 1.61) from the University of Portsmouth participated in exchange for course credit. The study employed a pretest-posttest between-subjects design; participants were randomly assigned to the disgust $(n=20)$ or the threat control $(n=20)$ condition. This study was part of a dissertation project. Therefore, the sample size obtained was based on practicalities around time and participants available.

\section{Materials and apparatus}

Tactile sensitivity (TS). TS was measured using Semmes-Weinstein monofilaments, thin pieces of plastic which are used to measure skin sensitivity in clinical settings (e.g., diabetics who have reduced blood flow; Kumar et al., 1991). The monofilaments consist of plastic rods with nylon fibers that vary in force (grams) from $0.008 \mathrm{~g}$ to $300 \mathrm{~g}$ (see Figure S1 in the Supplemental Material available online). The standardized procedure for measurement was followed (Schreuders, Slijper, \& Selles, 2010). The nylon fibers were applied with pressure to a patch of the participant's skin until it reached a ' $C$ ' shape. This procedure started with the smallest force $(0.008 \mathrm{~g})$ and was repeated with the monofilaments that have higher forces until the participant reported that they could feel the nylon fiber.

Stimuli. All participants were shown 20 neutral images (e.g., household furniture and appliances). Participants in the disgust condition were shown 20 disgust-evoking images (e.g., cadavers, faces, and vomit). Participants in the threat control condition were shown 20 threat-evoking images (e.g., dangerous animals, dangerous humans, and dangerous 
scenarios). All images were presented via a self-timed Microsoft PowerPoint presentation, with each image being displayed for $6 s$.

Box. For the TS measurements, a cardboard box was placed on a table, and a square hole was cut out at the participant's end with enough space for participants to place their forearm through. The experimenter's end was hooded to ensure that participants could not see the monofilaments being applied (see Figure S2).

\section{Procedure}

Participants were first asked to complete a short questionnaire that consisted of basic demographic questions, handedness, and information on nerve and skin conditions. A measurement was taken on the underside of the participant's non-dominant forearm in order to determine where the monofilaments should be applied. After this, participants were shown a Microsoft PowerPoint presentation of 20 affectively neutral images.

Participants were then seated at the table with the box and asked to place their nondominant arm with their palm facing up into the hole in order to take the pre-manipulation TS measure. Monofilaments were applied to approximately one-third way up the participant's non-dominant forearm. Each monofilament, from smallest to largest force, was applied in serial order. With each monofilament, participants were asked to report whether they felt something. If they had not, the experimenter moved onto the next monofilament.

Next, participants were asked to complete two tasks. The first was a questionnaire for an unrelated study on the themes of rejection and loneliness. The second was for participants to watch the PowerPoint presentation associated with their experimental condition. Participants then had their post-manipulation TS measure taken, following the same procedure as above.

\section{Results and discussion}


Figure 1 shows the mean pre- and post-manipulation TS scores. Pre-manipulation scores across conditions did not differ, $t(38)=0.17, p=.87$. To test the effect of the disgust manipulation on TS, an ANCOVA was conducted in which the pre-manipulation means were entered as a covariate and the post-manipulation means were entered as the dependent variable (there are various ways to analyze the data in this type of design, and the ANCOVA method has been recommended based on considerations of power and precision-see Vickers, 2001; Zhang et al., 2014). Although the pattern of results suggested greater sensitivity in the disgust condition, the analysis revealed no significant effect of condition, $F(1,37)=2.39, p=.13$, Cohen's $d=0.45$.

The results showed a nonsignificant trend in which TS increased for those in the disgust condition when compared to those in the threat control condition. However, the observed effect size suggests that the study may have been underpowered ( $N=76$ would be needed to achieve power of .80). In addition, this study had two key limitations. First, the experimenter was not blind to condition. Second, after each application of a monofilament, participants were prompted to decide whether they felt something or not. This introduces an element of signal detection, resulting in greater top-down influences than may be ideal for testing the hypothesis.

\section{Study 1}

Study 1 was conducted with several modifications. Two experimenters performed the experiment; an experimenter blind to the experimental condition measured tactile sensitivity. Participants were instructed to report unprompted when they felt something during the TS measure. Also, we measured individual differences in sensitivity to pathogen disgust, to examine potential moderating effects of trait disgust. Finally, different stimuli were used.

\section{Participants and design}


Eighty-one undergraduate students (63 women, 17 men, 1 undeclared; mean age = 20.15 years, $S D=5.06$ ) from the University of Bristol participated in exchange for course credit. Two additional participants were excluded from analysis because of errors during the study procedure. The study employed a pretest-posttest between-subjects design; participants were randomly assigned to the disgust $(n=41)$ or the control $(n=40)$ condition. On the basis of the effect size observed in the pilot study $(d=.45)$, a sample size of 81 yielded power of .82 .

\section{Materials and apparatus}

Stimuli. Participants in the disgust condition were exposed to a plastic container (dimensions $18 \mathrm{~cm} \times 12 \mathrm{~cm} \times 6 \mathrm{~cm}$ ) that was partly filled with live maggots of various colors. Maggots were used because they are ectoparasites specifically associated with disgust (rather than negative emotions more broadly). For purposes of hygiene, the maggots were replaced after every two days of testing. Maggots were stored in a fridge when not being used to minimize the odor in the testing room but were kept warm enough to be active during testing.

Participants in the control condition were exposed to the same type of container as in the disgust condition, but it contained approximately the same amount of uncooked basmati rice. Rice was used because of its visual resemblance to maggots while being affectively neutral (see Figure S3).

Both containers were shown without a lid to ensure that there was no physical barrier between the stimuli and the participants during exposure. Both containers had 23 adhesive gold sequin gems placed throughout the interior walls. This was used as part of a task to keep participants focused on the stimuli for a fixed amount of time (see below).

Screen. Two experimenters conducted the sessions. The first experimenter was in contact with the participants and presented either the control or disgust condition stimulus. The second experimenter, blind to condition, applied the monofilaments to measure TS at 
baseline and after the stimulus was presented. To ensure that the second experimenter remained blind to the condition of each participant, a cardboard self-standing screen with a square hole cut out at the bottom was used. The screen also ensured that participants could not see the monofilaments being applied (see Figure S4).

Pathogen disgust sensitivity. From the Three-Domain Disgust Scale (Tybur, Lieberman \& Griskevicius, 2009), seven items measuring pathogen disgust were used ( $\alpha=$ .62). Participants rated items such as "Stepping on dog poop", and ratings were made on a 4point scale (not at all disgusting, slightly disgusting, somewhat disgusting, very disgusting). Higher scores denoted greater disgust sensitivity.

\section{Procedure}

Participants were tested individually and seated in front of the cardboard screen. Both containers (the maggots and the rice) were placed into a crate where the participant and the second experimenter could not see the stimuli prior to the pre-manipulation measurement.

Participants removed any jewelry and placed their non-dominant arm to elbow level with their palm facing up through the hole. Participants were asked to look at a specific point on the wall in order to ensure that they would not receive any visual cues from any shadows cast by the second experimenter. Once the participant was ready, a visual signal was given by the first experimenter to the second experimenter to take the pre-manipulation measure of TS. Monofilaments were applied serially (with no prompting after each application), and participants were asked to inform the first experimenter when they felt something, who in turn gave a visual signal to the second experimenter to record which monofilament had been used.

Next, depending on condition, participants were presented with either a container of maggots or a container rice. The container was placed in a pre-selected area where the second experimenter was unable to see it. Participants counted the number of gold sequins in the 
container and reported the number to the first experimenter. If they completed this before $60 \mathrm{~s}$, they were requested to keep looking into the container until the time was up. After 60 s, the container was removed and TS was measured a second time.

Participants were asked not to emit any sounds upon seeing the maggots. However, in case of any vocalizations, the second experimenter wore headphones during the entirety of the experiment with an adequate amount of volume to block out any sound. Also, the first experimenter gave the same scripted instructions in both conditions to ensure that there were no other extraneous cues.

After the second TS measurement, participants were seated at a computer where they completed the pathogen disgust sensitivity scale and provided basic demographic data.

\section{Results and discussion}

Figure 2 shows the mean pre- and post-manipulation tactile sensitivity (TS) scores. Pre-manipulation scores across conditions did not differ significantly, $t(79)=1.70, p=.093$. To test the effect of the disgust manipulation on TS, an ANCOVA was conducted in which the pre-manipulation means were entered as a covariate. This analysis revealed a significant effect of condition with participants in the disgust condition showing lower TS scores (i.e., greater tactile sensitivity), $F(1,78)=16.54, p<.001, d=.57$.

We examined whether individual differences in pathogen disgust sensitivity played a role. Pathogen disgust was not significantly correlated with either pre- or post-manipulation TS means ( $p \mathrm{~s} \geq .064$ ). To assess whether disgust sensitivity moderated the effect of condition, we conducted a regression analysis in which pre-manipulation TS, condition, disgust sensitivity and condition $\times$ disgust sensitivity were simultaneously entered as predictors (with post-manipulation TS entered as the outcome variable). This analysis revealed no moderating effect of disgust sensitivity (condition $\times$ disgust sensitivity interaction effect $p=.17$ ). A visual inspection of the scatterplot showed that great pathogen 
disgust sensitivity was associated with higher TS scores (which is the opposite of what one might expect), and those in the disgust condition tended to have lower TS scores, particularly toward lower pathogen disgust scores.

These results more clearly demonstrated that participants in the disgust condition became more sensitive to touch when compared to neutral controls. Nevertheless, there were limitations in the design used. First, although our intention was to test the effect of visual perception of disgust-evoking stimuli, we cannot rule out the possibility that odor played a role. Also, lingering odor may have influenced participants in the neutral condition. Furthermore, because the control condition was affectively neutral, the results could be attributed to general negative arousal, rather than to disgust or disease concerns specifically. It was thus important to replicate this effect with a control condition that induced general negative affect.

\section{Study 2}

This study replicated the design of Study 1, with a couple of modifications. Instead of live maggots, we used a video of live maggots, which rules out any effects driven by odor. Different stimuli were used for this study in order to increase the generalizability of any further positive findings. As parasites are a form of disease threat, we also included a third physical threat condition, as a negative valence condition that is not associated with disease threat. Thus, a direct comparison could be made between a stimulus that evokes feelings of physical threat and one that evokes feelings of disease threat. A manipulation check was added to ensure that each set of stimuli evoked the appropriate emotion. Due to the null results found in Study 1 involving individual differences in pathogen disgust sensitivity, this measure was removed for Study 2.

\section{Participants and Design}


Two hundred and eighteen undergraduate and postgraduate students (159 females, 56 males, 3 undeclared; mean age $=22.36$ years, $S D=6.52$ ) from the University of Bristol participated in exchange for course credit or on a voluntary basis. The study employed a pretest-posttest between-subjects design; participants were randomly assigned to the disgust ( $n=72)$, threat $(n=73)$, or neutral $(n=73)$ condition. The focal comparisons were between the disgust condition and each of the other two conditions. The previous two studies showed roughly similar effect sizes ( $d=.45$ and .57 , respectively). Using the more conservative effect size value of .45 , the current study had power of approximately .97 to detect the key effects.

\section{Materials and Apparatus}

Stimuli. The stimuli for the manipulation were presented on a Lenovo 10.1" Tablet. In the disgust condition, participants viewed a 60s video of maggots; in the threat control, they viewed a 60s clip of the Baltimore riots (the riot clip was specifically selected as it displayed people being trapped with the windows being smashed from the outside, likely to evoke fear, but not disgust). In the neutral condition, participants looked at a pin on the wall for $60 \mathrm{~s}$.

Manipulation check. Five items were used to assess what emotions participants felt toward the stimulus that was presented. The items were angry, happy, disgusted, sad, and threatened, which were measured on a 5 -point scale $(1=$ not at all, and $5=$ very much $)$.

\section{Results and discussion}

Table 1 shows the manipulation check mean scores and SDs. One-way ANOVAs were conducted on the disgust and threat ratings in order to investigate whether each condition evoked the appropriate emotion. Results for disgust ratings revealed a significant effect of condition, $F(2,212)=75.93, p<.001$; Bonferroni pairwise comparisons revealed that disgust ratings were higher in the disgust condition when compared to neutral $(p<.001)$ and threat $(p<.05)$ conditions. Results for threat ratings revealed a significant effect of 
condition, $F(2,212)=19.55, p<.001$; Bonferroni pairwise comparisons revealed disgust ratings were higher in the threat condition when compared to neutral $(p<.001)$ and threat $(p$ $<.001)$ conditions.

Figure 3 shows the mean pre- and post-manipulation tactile sensitivity (TS) values. A one-way ANOVA on the pre-manipulation TS values showed no statistical differences between the three conditions $(p=.57)$. To test the effect of the disgust manipulation on TS, an ANCOVA was conducted in which the pre-manipulation means were entered as a covariate. This analysis revealed a significant effect of condition, $F(2,214)=21.21, p<.001$. Bonferroni pairwise comparisons were conducted to probe the differences between the three conditions. Post-manipulation TS values were lower in the disgust condition when compared to neutral $(p<.001, d=0.90)$ and threat $(p<.001, d=0.94)$ conditions. There was no difference between the threat and neutral conditions $(p>.99, d=0.09)$.

These results extend those of the earlier studies. Participants in the disgust condition showed greater tactile sensitivity when compared to those in the neutral and threat control conditions. The method used in Study 2 warrants the interpretation that the effect occurred via mere visual perception and cannot be attributed to general negative arousal.

\section{General discussion}

Across three studies, we tested the hypothesis that disgust may increase tactile sensitivity. The results of the pilot study revealed a pattern in the hypothesized direction. Studies 1 and 2 showed that disgust did produce greater TS when compared to both neutral and threat controls. Given the methods used, the results lend confidence to the interpretation that greater TS was specifically due to disgust rather than general negative arousal. These findings, to our knowledge, are the first to show evidence that evoking disgust can cause changes in skin sensitivity. 
Our findings are consistent with the broader logic of disease avoidance (Curtis, de Barra, \& Aunger, 2011; Murray \& Schaller, 2016; Schaller \& Park, 2011), providing evidence that perceptual sensitivity is flexibly altered in order to facilitate faster detection of disease threats that may come into contact with the skin. These findings demonstrate how visually received perceptual cues associated with disease threat can activate an immediate functional physiological response. It would appear that conceptual information associated with disease threat creates top-down influences that affect TS, which is consistent with previous findings demonstrating that top-down influences alter tactile perception (Spence \& Gallace, 2007). Specifically, these top-down influences seem to have affected the threshold for tactile detection of stimuli. This is similar to recent findings showing the effect of disgust on olfactory thresholds (Chan, Holland, van Loon, Arts, \& van Knippenberg, 2016).

Future studies might investigate whether more indirect cues to disease threat could have the same effect on tactile sensitivity. Previous research has shown that physical anomalies that are objectively harmless (e.g., physical disability) can activate diseaseavoidance responses, such as physical distancing (Park, Faulkner, \& Schaller, 2003) and stigmatization (Kurzban \& Leary, 2001). If such physical features also cause changes in tactile sensitivity, that would further clarify the role of disease avoidance in negative perceptions of physical anomalies. As recent evidence has suggested that cues associated with skin-transmitted parasites may form a subcategory of disgust elicitors (Blake, Yih, Zhao, Sung, \& Harmon-Jones, 2016), further studies may investigate whether similar increases in TS can be observed when disgust-evoking stimuli, not associated with contamination via the skin, are used.

Future studies could also help increase our understanding of psychological mechanisms associated with extreme self-grooming. Trichotillomania is a compulsive control disorder that is characterized by excessive hair pulling to the point of alopecia (baldness). 
Contamination-related obsessive compulsive disorder is characterized by obsessive thoughts of contamination fear that lead to compulsive rituals of excessive self-grooming. This excessive self-grooming, designed to neutralize the fear of contamination, can lead to splitting skin, extreme red soars, and swelling. It is possible that those who suffer from these conditions have lower tactile thresholds. Thus, findings from future research on the links between disease-avoidance processes and tactile sensitivity could have therapeutic applications. 


\section{References}

Autino, B., Noris, A., Russo, R., \& Castelli, F. (2012). Epidemiology of malaria in endemic areas. Mediterranean Journal of Hematology and Infectious Diseases, 4, 2012060. doi: 10.4084/MJHID.2012.060

Bell, J. F., Jellison, W. L., \& Owen, C. K. (1962). Effects of limb disability on lousiness in mice. I. Preliminary studies. Experimental parasitology, 12, 176-183. doi: $10.1016 / 0014-4894(62) 90055-3$

Bennett, G. F. (1969). Boophilus microplus (Acarina: Ixodidae): Experimental infestations on cattle restrained from grooming. Experimental Parasitology, 26, 323-328. doi: $10.1016 / 0014-4894(69) 90125-8$

Blake, K. R., Yih, J., Zhao, K., Sung, B., \& Harmon-Jones, C. (2016). Skin-transmitted pathogens and the heebie jeebies: evidence for a subclass of disgust stimuli that evoke a qualitatively unique emotional response. Cognition and Emotion, 1-16. doi:10.1080/02699931.2016.1202199

Bolles, R. C. (1960). Grooming behavior in the rat. Journal of comparative and physiological psychology, 53, 306. doi: 10.1037/h0045421

Carter, R., \& Mendis, K. N. (2002). Evolutionary and historical aspects of the burden of malaria. Clinical microbiology reviews, 15, 564-594. doi:

10.1128/CMR.15.4.564.2002

Chan, K. Q., Holland, R. W., van Loon, R., Arts, R., \& van Knippenberg, A. (2016). Disgust and fear lower olfactory threshold. Emotion, 16, 740. doi:10.1037/emo0000113

Clark, J. A., \& Fessler, D. M. T. (2014). Recontextualizing the behavioral immune system within psychoneuroimmunology. Evolutionary Behavioral Sciences, 8, 235-243. doi:10.1037/ebs0000024 
Curtis, V., de Barra, M., \& Aunger, R. (2011). Disgust as an adaptive system for disease avoidance behavior. Philosophical Transactions of the Royal Society B, 366, 389401. doi:10.1098/rstb.2010.0117

Gonçalves, M. L. C., Araújo, A., \& Ferreira, L. F. (2003). Human intestinal parasites in the past: new findings and a review. Memórias do Instituto Oswaldo Cruz, 98, 103-118. doi: 10.1590/S0074-02762003000900016

Graczyk, T. K., Knight, R., Gilman, R. H., \& Cranfield, M. R. (2001). The role of non-biting flies in the epidemiology of human infectious diseases. Microbes and Infection, 3, 231-235. doi: 10.1016/S1286-4579(01)01371-5

Hart, B. L. (1990). Behavioral adaptations to pathogens and parasites: Five strategies. Neuroscience \& Biobehavioral Reviews, 14, 273-294.

Hart, L. A., \& Hart, B. L. (1988). Autogrooming and social grooming in impala. Annals of the New York Academy of Sciences, 525, 399-402. doi: 10.1111/j.17496632.1988.tb38625.x

Hosoi, J. (2006). Stress and the skin. International Journal of Cosmetic Science, 28, 243-246.

Kumar, S., Fernando, D. J. S., Veves, A., Knowles, E. A., Young, M. J., \& Boulton, A. J. M. (1991). Semmes-Weinstein monofilaments: a simple, effective and inexpensive screening device for identifying diabetic patients at risk of foot ulceration. Diabetes Research and Clinical Practice, 13, 63-67.

Kurzban, R., \& Leary, M. R. (2001). Evolutionary origins of stigmatization: The functions of social exclusion. Psychological Bulletin, 127, 187-208. doi:10.1037//00332909.127.2.187

Madison, K. C. (2003). Barrier function of the skin: "la raison d'être" of the epidermis. Journal of Investigative Dermatology, 121, 231-241. 
Miller, S. L., \& Maner, J. K. (2011). Sick body, vigilant mind: The biological immune system activates the behavioral immune system. Psychological Science, 22, 1467-1471. doi:10.1177/0956797611420166

Murray, D. R., \& Schaller, M. (2016). The behavioral immune system: Implications for social cognition, social interaction, and social influence. Advances in Experimental Social Psychology, 53, 75-129. doi:10.1016/bs.aesp.2015.09.002

Oaten, M., Stevenson, R, J., \& Case, T. I. (2009). Disgust as a disease-avoidance mechanism. Psychological Bulletin, 135, 303-321. doi:10.1037/a0014823

Park, J. H., Faulkner, J., \& Schaller, M. (2003). Evolved disease-avoidance processes and contemporary anti-social behavior: Prejudicial attitudes and avoidance of people with physical disabilities. Journal of Nonverbal Behavior, 27, 65-87. doi:10.1023/A:1023910408854

Parham, P. (2009). The immune system (3rd ed.). New York: Garland Science.

Prokop, P., \& Fedor, P. (2013). The effects of parasites on human behaviour: An evolutionary perspective. Problems of Psychology in the 21st Century, 5, 46-64.

Prokop, P., Fančovičová, J., \& Fedor, P. (2014). Parasites enhance self-grooming behaviour and information retention in humans. Behavioural Processes, 107, 42-46.

de Roode, J. C., \& Lefèvre, T. (2012). Behavioral immunity in insects. Insects, 3, 789-820. doi: 10.3390/insects3030789

Schaller, M. (2011). The behavioural immune system and the psychology of human sociality. Philosophical Transactions of the Royal Society B: Biological Sciences, 366, 34183426. doi:10.1098/rstb.2011.0029

Schaller, M., Miller, G. E., Gervais, W. M., Yager, S., \& Chen, E. (2010). Mere visual perception of other people's disease symptoms facilitates a more aggressive immune response. Psychological Science, 21, 649-652. doi:10.1177/0956797610368064 
Schaller, M., \& Park, J. H. (2011). The behavioral immune system (and why it matters). Current Directions in Psychological Science, 20, 99-103. doi:10.1177/0963721411402596

Schreuders, T., Slijper, H., \& Selles, R. [Ruud Seles]. (2010, August 17). Sensibility testing of the hand using Semmes and Weinstein [Video file]. Retrieved from https://www.youtube.com/watch?v=ufKRkUqvNQk

Spence, C., \& Gallace, A. (2007). Recent developments in the study of tactile attention. Canadian Journal of Experimental Psychology/Revue canadienne de psychologie expérimentale, 61, 196-207.

Stevenson, R. J., Hodgson, D., Oaten, M. J., Barouei, J., \& Case, T. I. (2011). The effect of disgust on oral immune function. Psychophysiology, 48, 900-907. doi:10.1111/j.1469-8986.2010.01165.x

Stevenson, R. J., Hodgson, D., Oaten, M. J., Moussavi, M., Langberg, R., Case, T. I., \& Barouei, J. (2012). Disgust elevates core body temperature and up-regulates certain oral immune markers. Brain, Behavior, and Immunity, 26, 1160-1168. doi:10.1016/j.bbi.2012.07.010

Tranter, C., Lefèvre, L., Evison, S. E., \& Hughes, W. O. (2014). Threat detection: contextual recognition and response to parasites by ants. Behavioral Ecology, aru203. doi: 10.1093/beheco/aru203

Tybur, J. M., Lieberman, D., \& Griskevicius, V. (2009). Microbes, mating, and morality: Individual differences in three functional domains of disgust. Journal of Personality and Social Psychology, 97, 103-122. doi:10.1037/a0015474

Vickers, A. J. (2001). The use of percentage change from baseline as an outcome in a controlled trial is statistically inefficient: A simulation study. BMC Medical Research Methodology, 1, 6. doi:10.1186/1471-2288-1-6 
Williams, I. R., \& Kupper, T. S. (1996). Immunity at the surface: homeostatic mechanisms of the skin immune system. Life sciences, 58(18), 1485-1507. doi: 10.1016/00243205(96)00042-2

Wolfe, N. D., Dunavan, C. P., \& Diamond, J. (2007). Origins of major human infectious diseases. Nature, 447, 279-283. doi:10.1038/nature05775

Zhang, S., Paul, J., Nantha-Aree, M., Buckley, N., Shahzad, U., Cheng, J., DeBeer, J., Winemaker, M., Wismer, D., Puntakee, D., Avram, V., \& Thabane, L. (2014). Empirical comparison of four baseline covariate adjustment methods in analysis of continuous outcomes in randomized controlled trials. Clinical Epidemiology, 6, 227235. doi:10.2147/CLEP.S56554 


\section{Footnotes}

1. There is an intuitive and sensible argument to say that scratching the skin would breach this protective barrier and increase the disease threat, rather than decrease it. As with most behaviors, there is a costs/benefits trade off. For scratching, the costs of breaching the skin barrier could possibly be off set against the costs of allowing the disease threat to contaminate the individual. Furthermore, superficial scratching may cause removal of the uppermost layers on the skin, but will rarely actually be fully breached. 
Table 1

Mean Scores (and SDs) on the Manipulation Check Items $(1=$ not at all, $5=$ very much)

\begin{tabular}{llllll}
\hline & Angry & Happy & Disgusted & Sad & Threatened \\
\hline $\begin{array}{l}\text { Wall (neutral) } \\
\text { condition }\end{array}$ & $1.09(0.38)$ & $1.32(0.72)$ & $1.11(0.43)$ & $1.16(0.44)$ & $1.32(0.62)$ \\
$\begin{array}{l}\text { Riot (threat) } \\
\begin{array}{l}\text { condition } \\
\text { Maggots (disgust) }\end{array}\end{array}$ & $2.29(1.02)$ & $1.14(0.42)$ & $2.58(1.14)$ & $2.71(1.20)$ & $2.33(1.12)$ \\
\begin{tabular}{l} 
condition \\
\hline
\end{tabular} & $1.19(0.62)$ & $1.22(0.51)$ & $3.04(1.19)$ & $1.33(0.68)$ & $1.64(0.94)$ \\
\hline
\end{tabular}

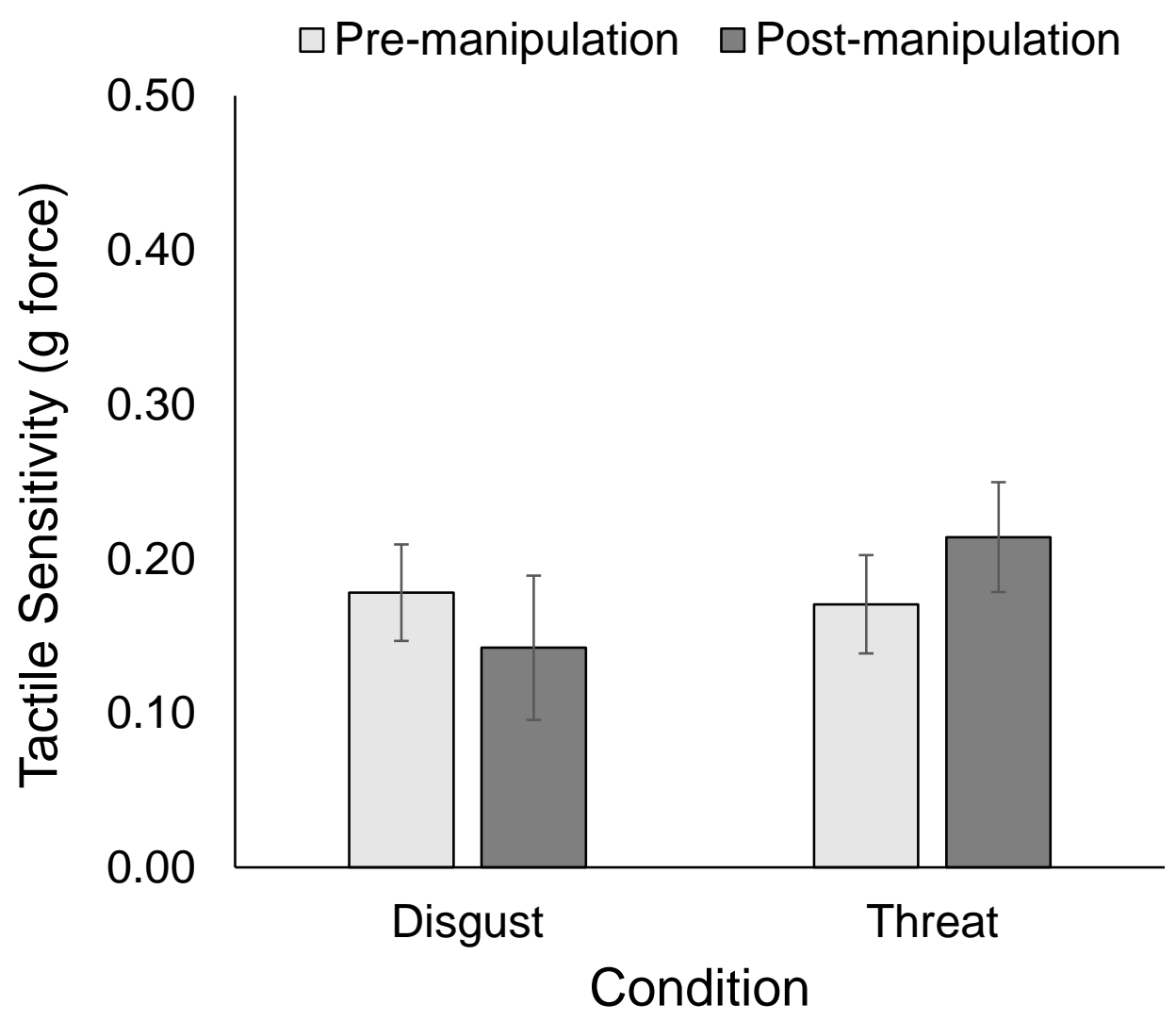

Figure 1. Mean pre- and post-manipulation tactile sensitivity values for each condition in the Pilot study; lower values indicate greater tactile sensitivity (error bars denote standard errors). 


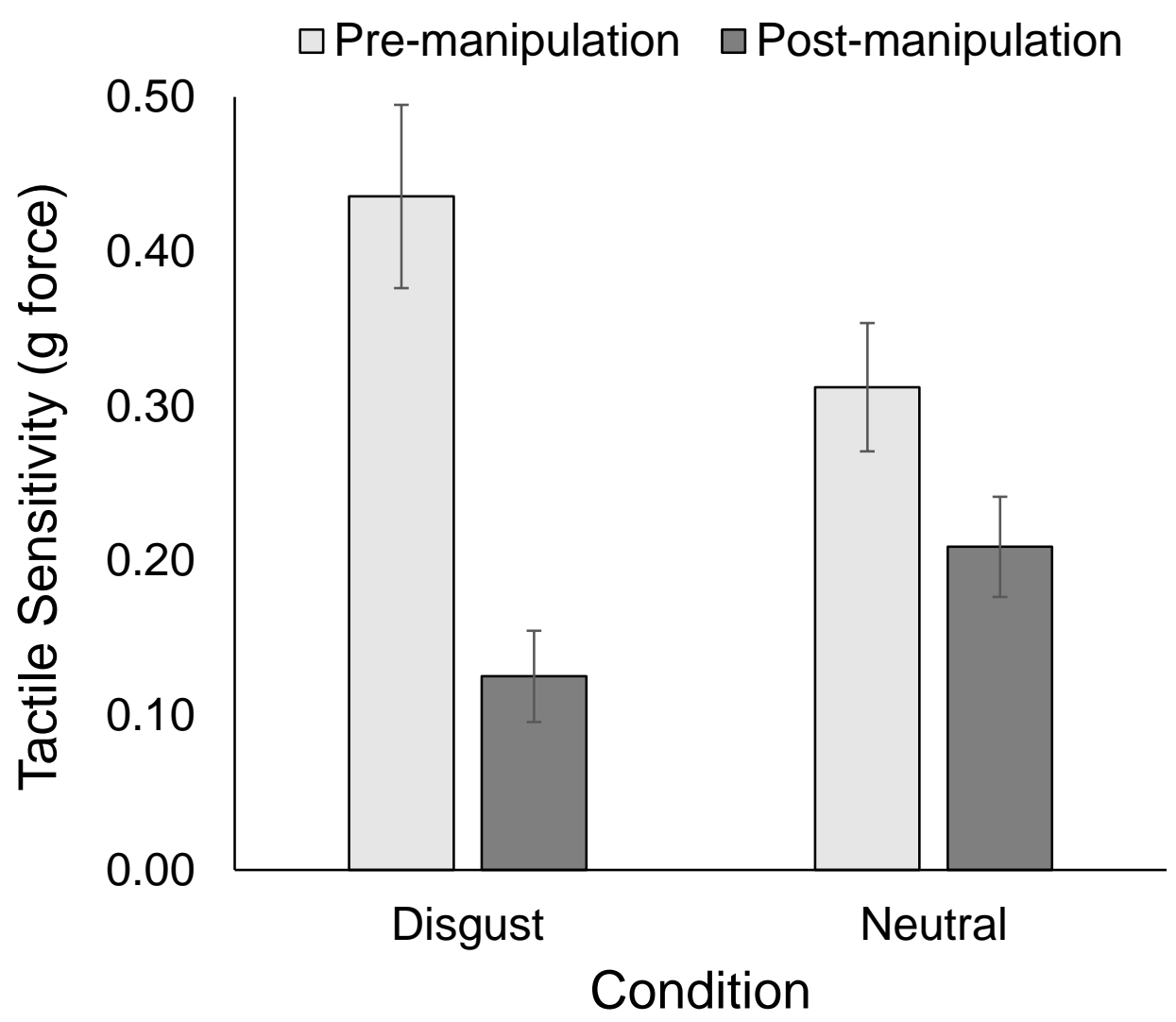

Figure 2. Mean pre- and post-manipulation tactile sensitivity values for each condition in Study 1; lower values indicate greater tactile sensitivity (error bars denote standard errors). 


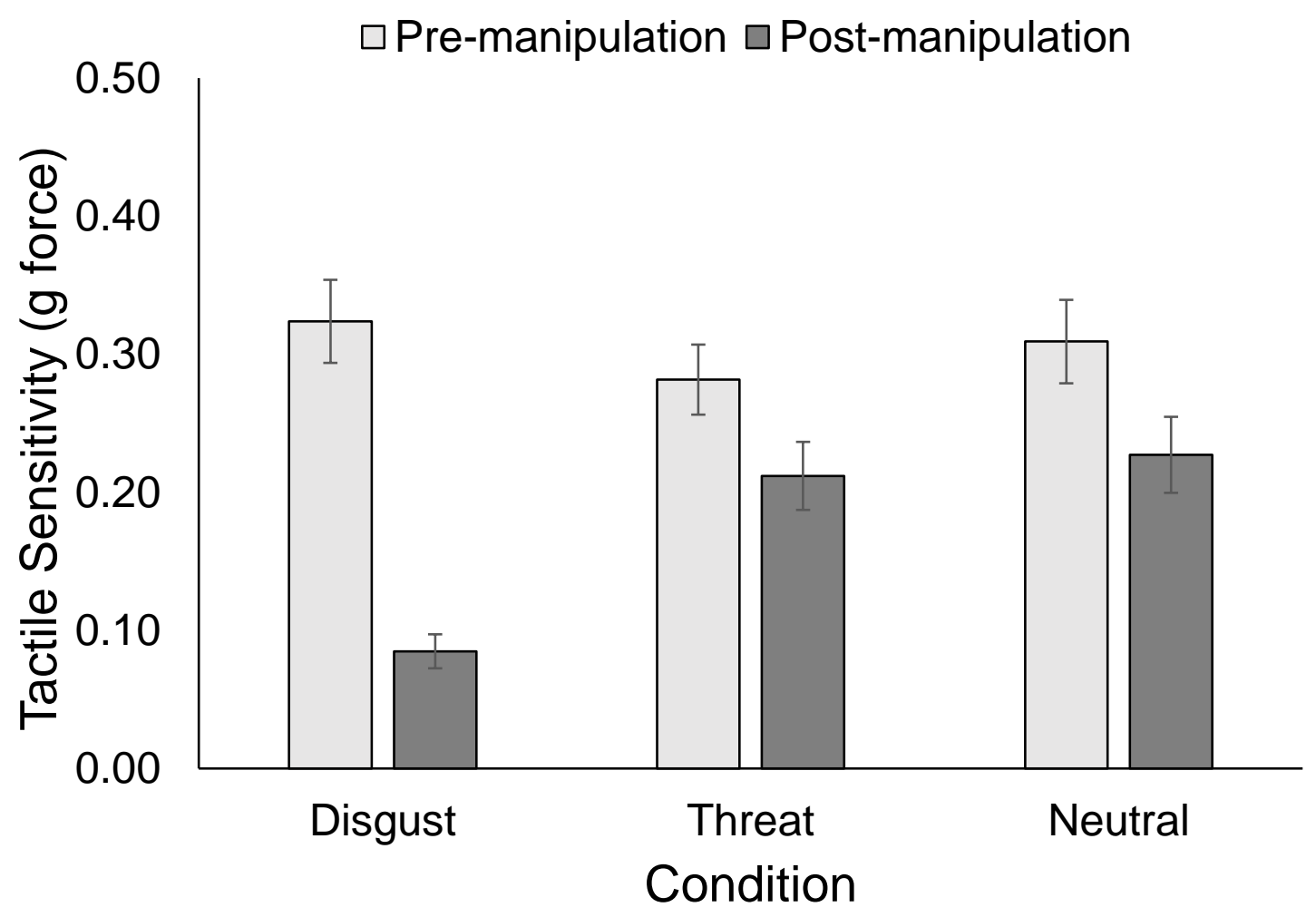

Figure 3. Mean pre- and post-manipulation tactile sensitivity values for each condition in Study 2; lower values indicate greater tactile sensitivity (error bars denote standard errors). 


\section{Supplemental Online Material}

Hunt et al. Making your skin crawl: The role of tactile sensitivity in disease avoidance.

Biological Psychology.

Figure S1. A photographic image of the monofilaments used to measure tactile sensitivity

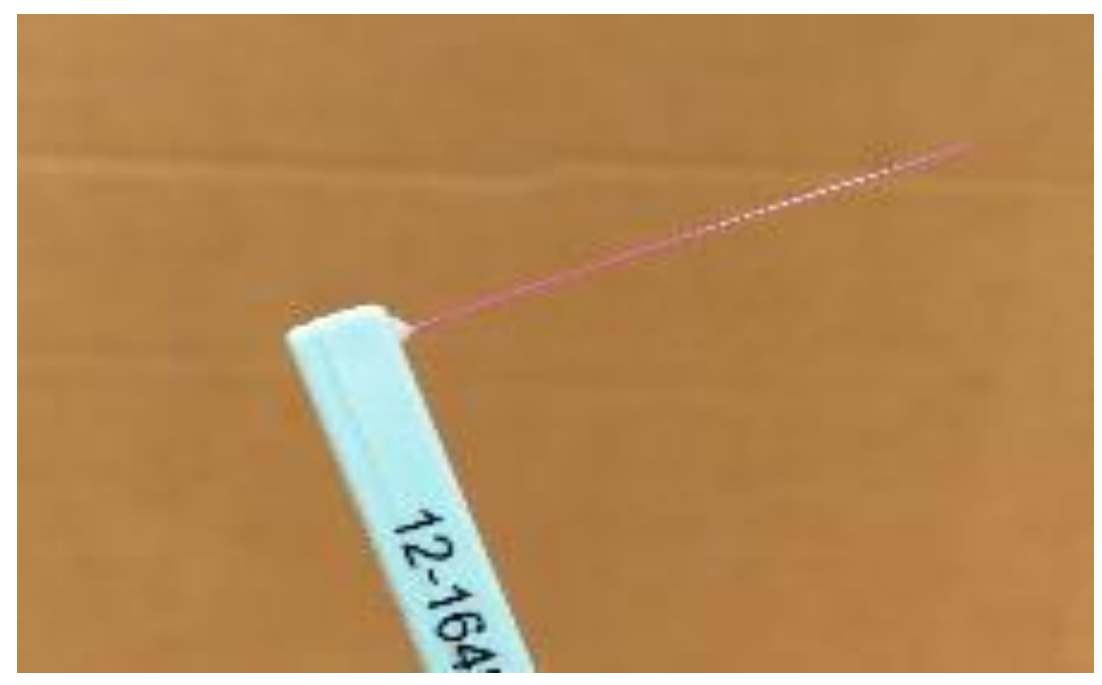

Figure S2. A photographic image of the box used in the pilot study

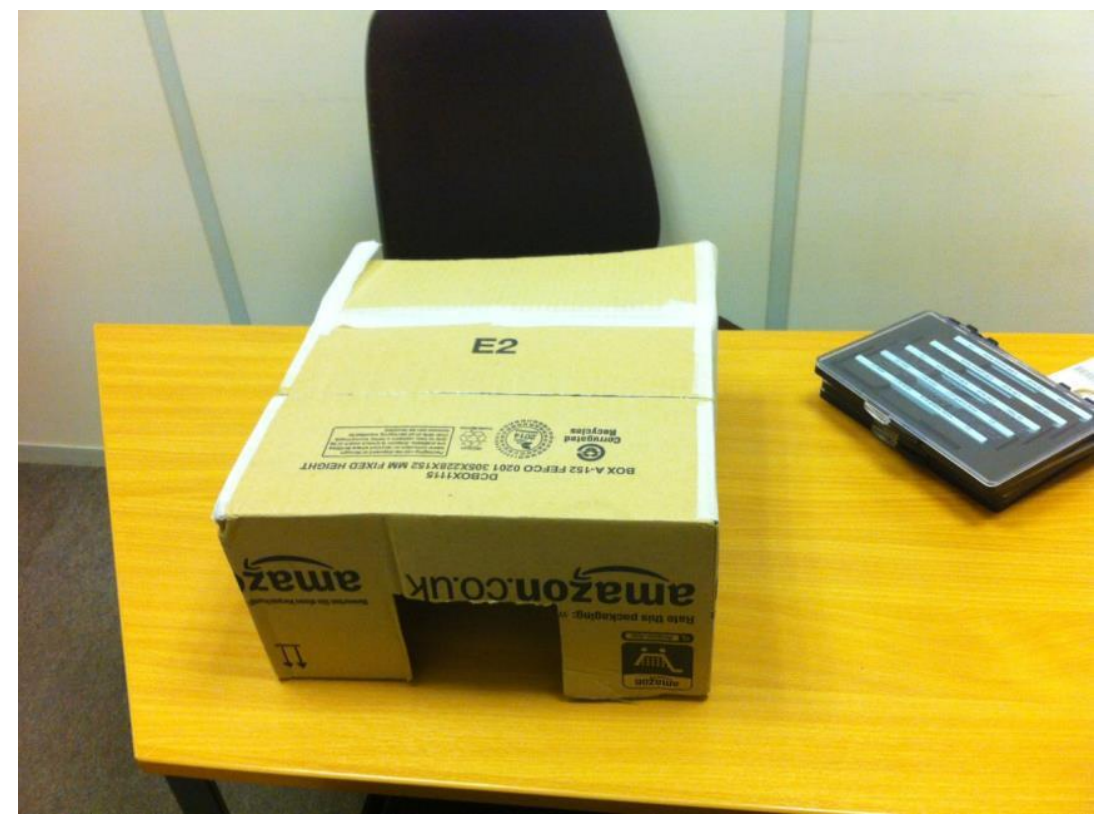


Figure S3. A photographic image of the stimuli presented in study 1

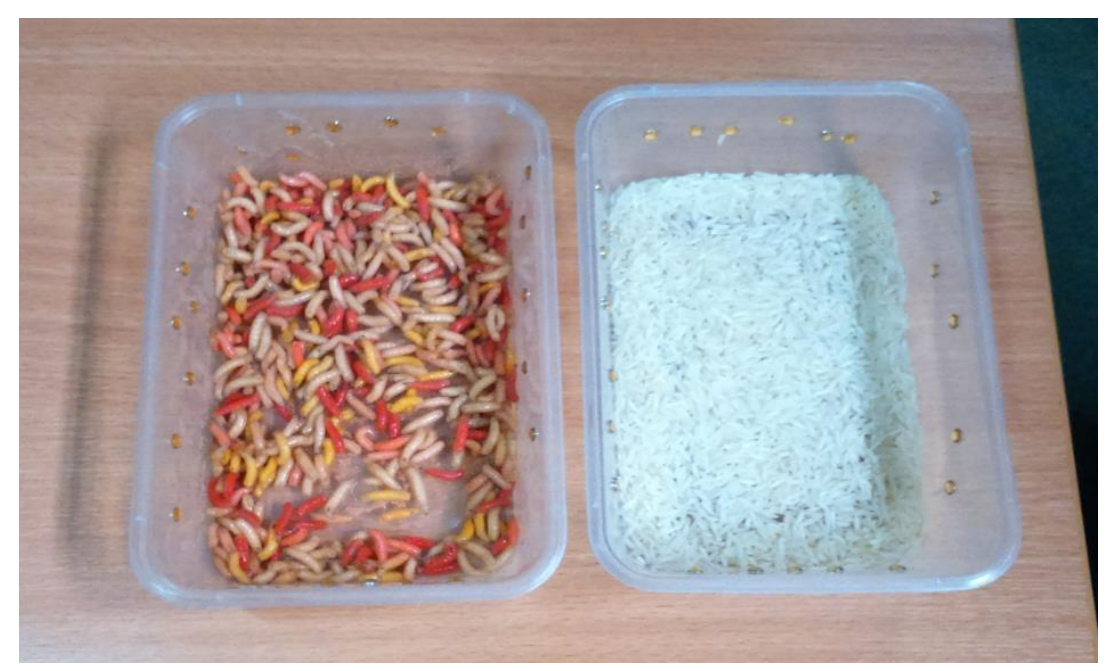

Figure S4. A photographic image of the screen used in studies 1 and 2

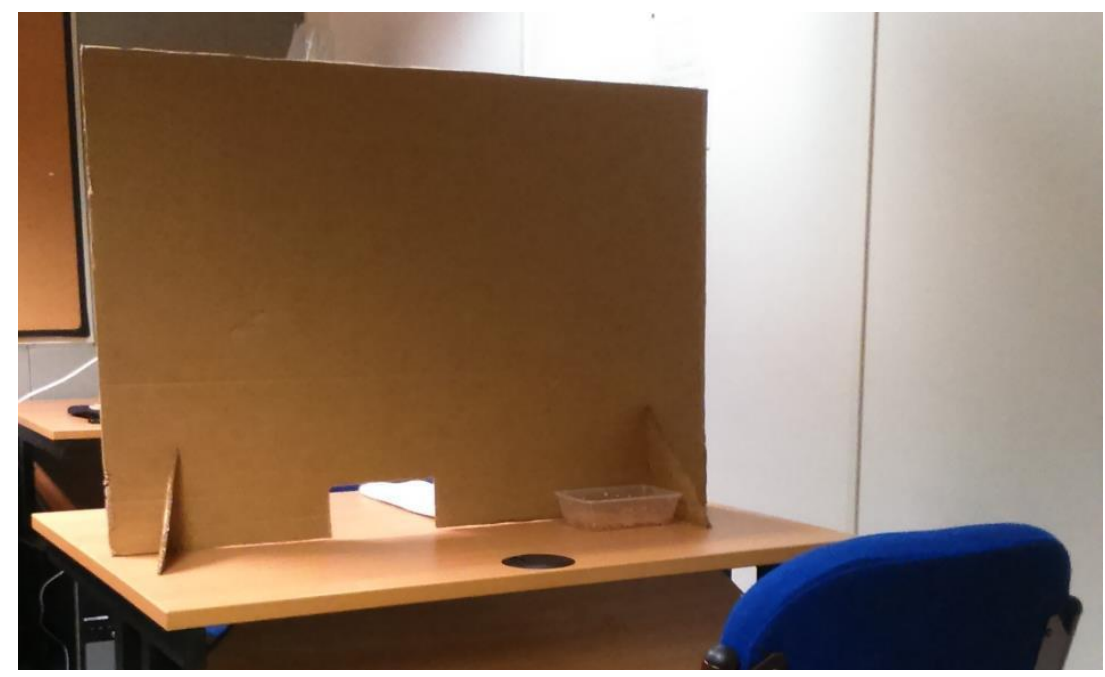




\section{Highlights}

- Evoking disgust increases tactile sensitivity (TS).

- This function has evolved to locate disease threat more quickly.

- Findings are specifically related to disgust as threat did not increase TS.

- These, to our knowledge, are the first findings to link disgust and TS. 